\title{
Extreme Adaptive Optics for the Thirty Meter Telescope
}

Bruce Macintosh, et al.

May 15, 2006

Astronomical Telescopes and Instrumentation 2006 orlando, FL, United States

May 24, 2006 through May 31, 2006 
This document was prepared as an account of work sponsored by an agency of the United States Government. Neither the United States Government nor the University of California nor any of their employees, makes any warranty, express or implied, or assumes any legal liability or responsibility for the accuracy, completeness, or usefulness of any information, apparatus, product, or process disclosed, or represents that its use would not infringe privately owned rights. Reference herein to any specific commercial product, process, or service by trade name, trademark, manufacturer, or otherwise, does not necessarily constitute or imply its endorsement, recommendation, or favoring by the United States Government or the University of California. The views and opinions of authors expressed herein do not necessarily state or reflect those of the United States Government or the University of California, and shall not be used for advertising or product endorsement purposes. 


\title{
Extreme Adaptive Optics for the Thirty Meter Telescope
}

\author{
Bruce Macintosh $^{*}{ }^{\text {ab }}$, Mitchell Troy ${ }^{\text {ac }}$, Rene Doyon ${ }^{\mathrm{d}}$, James Graham ${ }^{\mathrm{ae}}$, Kevin Baker ${ }^{\mathrm{ab}}$, Brian \\ Bauman $^{\mathrm{ab}}$, Christian Marois $^{\mathrm{ab}}$, David Palmer ${ }^{\mathrm{ab}}$, Donald Phillion ${ }^{\mathrm{ab}}$, Lisa Poyneer ${ }^{\mathrm{ab}}$, Ian Crossfield ${ }^{\mathrm{ac}}$, \\ Philip Dumont $^{\text {ac }}$, B. Marty Levine ${ }^{\text {ac }}$, Michael Shao ${ }^{\text {ac }}$, Gene Serabyn ${ }^{\text {ac }}$, Chris Shelton ${ }^{\text {ac }}$, Gautum \\ Vasisht $^{\text {ac }}$, James K. Wallace ${ }^{\text {ac }}$, Jean-Francois Lavigne ${ }^{d}$, Philippe Valee ${ }^{d}$, Neil Rowlands ${ }^{\mathrm{f}}$, Ken Tam ${ }^{\mathrm{f}}$, \\ Daniel Hackett ${ }^{\mathrm{f}}$ \\ ${ }^{a}$ NSF Center for Adaptive Optics \\ ${ }^{b}$ Lawrence Livermore National Laboratory, 7000 East Ave., Livermore, CA 94551 \\ ${ }^{\mathrm{c} J e t}$ Propulsion Laboratory, California Institute of Technology, Pasadena, CA 91109 \\ ${ }^{d}$ Universite de Montreal, Department de Physique, Montreal, QC, H3C 3J7, Canada \\ ${ }^{\mathrm{e}}$ Department of Astronomy, University of California at Berkeley, Berkeley, CA 94720

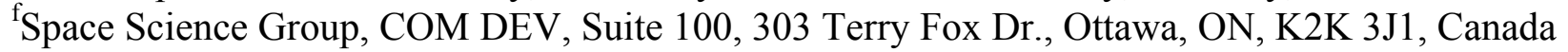

\begin{abstract}
Direct detection of extrasolar Jovian planets is a major scientific motivation for the construction of future extremely large telescopes such as the Thirty Meter Telescope (TMT). Such detection will require dedicated high-contrast AO systems. Since the properties of Jovian planets and their parent stars vary enormously between different populations, the instrument must be designed to meet specific scientific needs rather than a simple metric such as maximum Strehl ratio. We present a design for such an instrument, the Planet Formation Imager (PFI) for TMT. It has four key science missions. The first is the study of newly-formed planets on 5-10 AU scales in regions such as Taurus and Ophiucus this requires very small inner working distances that are only possible with a $30 \mathrm{~m}$ or larger telescope. The second is a robust census of extrasolar giant planets orbiting mature nearby stars. The third is detailed spectral characterization of the brightest extrasolar planets. The final targets are circumstellar dust disks, including Zodiacal light analogs in the inner parts of other solar systems. To achieve these, PFI combines advanced wavefront sensors, high-order MEMS deformable mirrors, a coronagraph optimized for a finely- segmented primary mirror, and an integral field spectrograph.
\end{abstract}

Keywords: Adaptive optics, extremely large telescopes, coronagraphs, extrasolar planets

\section{INTRODUCTION AND SCIENCE MOTIVATION}

Precision radial velocity measurements have now yielded the discovery of over 160 planets. Direct imaging of these planets, as opposed to detection of the effects of orbital motion on their parent star, is now feasible, and the first young planet in a wide orbit may have been detected using adaptive optics systems ${ }^{1}$. Gemini and the VLT are building the first generation of high contrast "extreme" adaptive optics (ExAO) systems, which deliver planet-imaging performance at separations $>0.1$ arcseconds. These systems will make the first surveys of the outer regions of solar systems by detecting the self-luminous radiation of young planets (1-10 $\left.\mathrm{M}_{\mathrm{J}}, 10-1000 \mathrm{MYr}\right)$. The 8-m ExAO systems cannot see close enough to the host stars to image Doppler-detected or other mature planets in reflected light, and they cannot reach the relatively distant, young clusters and associations where planets are likely to still be forming. The Planet Formation Instrument will use the nearly four-fold improved angular resolution of TMT to peer into the inner solar systems of planet bearing stars to yield a unified sample of planets with known Keplerian orbital elements and atmospheric properties. In star formation regions, where T Tauri stars (young solar type stars) are found in abundance, PFI can see into the snow line, where the icy cores of planets like Jupiter must have formed. Thus, TMT could be the first facility to witness the formation of new planets directly. Because of the short lifetimes relative to the Galactic star formation rate, young planet-forming systems are rare and found in significant numbers only in distant $(>150 \mathrm{pc})$ star forming clouds. The inner working distance required to study planet formation in situ is therefore of order 35 milli arc seconds (5 AU at 150 pc). Since a typical coronagraph has an inner working distance $>3-5 \lambda / D$, It is evident that the TMT will be the first

\footnotetext{
*bmac@igpp.llnl.gov
} 
facility to enable direct observation of planets emerging from their parent discs. Likewise, to detect nearby mature planets in reflected light, a comparable angular resolution will be needed ( 30 mas $=0.3 \mathrm{AU}$ at $10 \mathrm{pc})$. TMT will thus also be the first facility to be able to directly detect a sizable number of reflected light Jovian planets. The unique combination of angular resolution and sensitivity of TMT will thus enable direct images and spectra to be obtained for both young and old planets (Figure 1). The main instrumental capabilities needed to take advantage of the TMT in this regard is high contrast imaging at an angular separation of a few $\lambda / \mathrm{D}$ from bright stars in the near infrared. This goal is the basic driver for the instrument described here, the Planetary Formation Instrument (PFI).

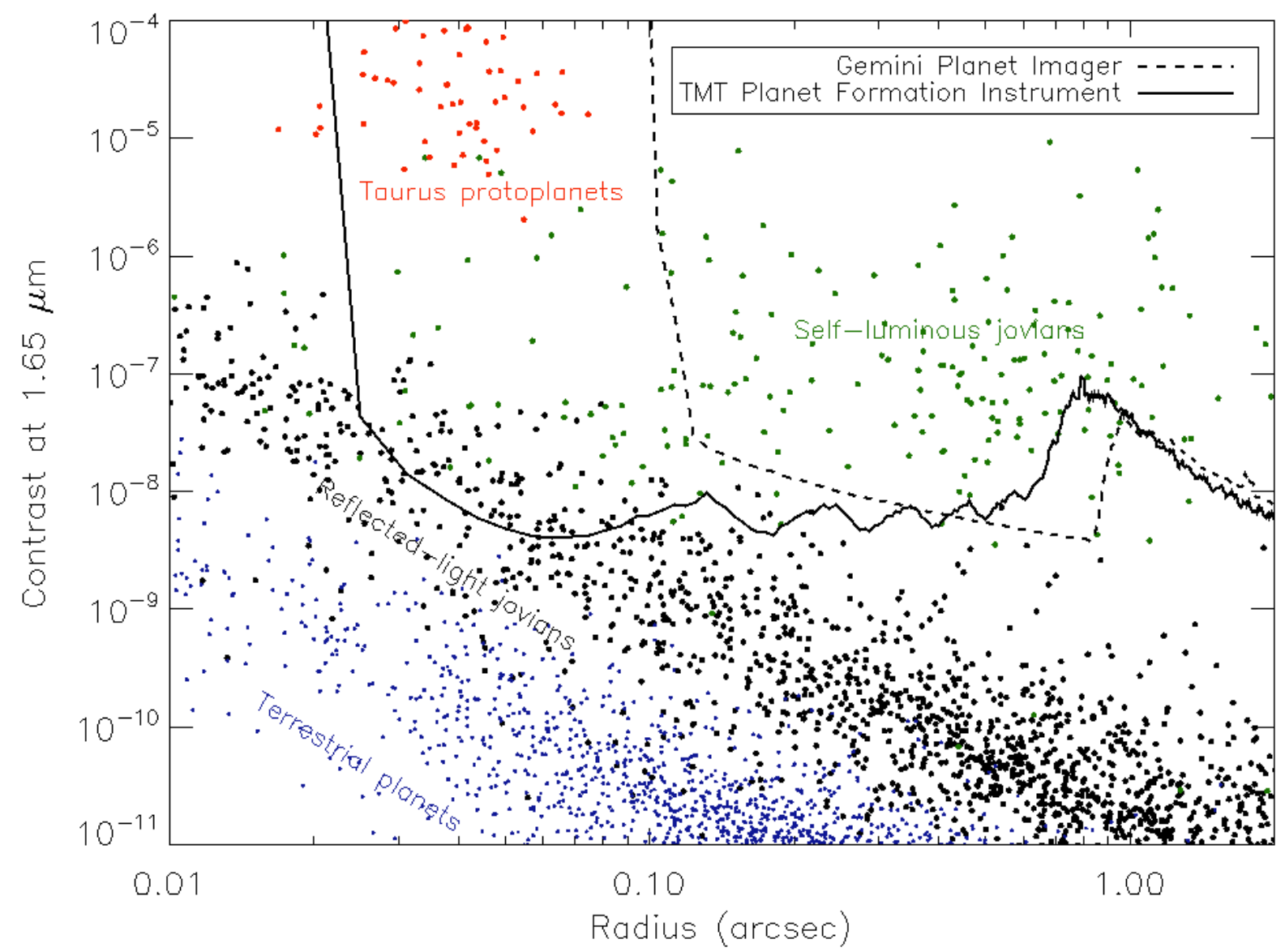

Figure 1: Contrast-separation plot for a Monte Carlo simulation of a variety of targets in the solar neighborhood. Blue dots are rocky planets, beyond the reach of even TMT. Black dots are mature Jovian planets reflecting sunlight. Green dots are self-luminuous Jovian planets, typically those with masses of 3-10 Jupiter masses and ages $<1$ Gyr. Red dots are extremely young planets, recently formed or still accreting, e.g. in the Taurus star-forming region. The expected sensitivity of PFI and the Gemini Planet Imager for a bright ( $4^{\text {th }}$ magnitude) target are overlaid.

\section{OVERALL ARCHITECTURE}

The scientific goals of PFI lead to extremely challenging technical requirements (Table 1). Current AO systems achieve contrasts on the order of $10^{-5}$ at angular separations of $\sim 1$ arc second; TMT PFI requires a three order of magnitude improvement in contrast and a factor of twenty in angular separation. Some of the improvement of course comes from the larger telescope aperture, but equally important is the design of an AO system and instrument dedicated to high contrast imaging, with precise and accurate control of optical wavefronts. The combined requirements of high dynamic range, a wide variety of target brightnesses, very high angular resolution and the need to minimize systematic errors, 
lead to a multi-stage integrated instrument with each subsystem serving a well-defined role in controlling a particular aspect of the problem.

The high-speed front AO system (Section 3)is optimized for searching for planets orbiting nearby field stars. This requires achieving extremely high contrast $\left(>10^{-8}\right)$ on bright targets, which in turn requires very high update rates $(2-4$ $\mathrm{kHz}$ ) to minimize dynamic atmospheric errors. Achieving good wavefront correction at these rates will requirefficient use of the available photons. To achieve this, we have selected as our baseline a variant of the pyramid wavefront sensor run in a quasi-interferometric mode. This takes advantage of the high Strehl ratio at the wavefront sensing wavelength to achieve measurement errors a factor of 2-4 better than a conventional Shack-Hartmann sensor. Combined with a compact, high-order MEMS DM this system will produce H-band Strehl ratios above 0.9 on bright stars and 0.84 down to $I=9$ mag. On dimmer stars, it will provide partial correction to enable the back (interferometric) wavefront sensor to provide the bulk of the wavefront correction.

Table 1: PFI Requirements. Speckle suppression processing of IFS data cubes is expected to increase contrast by a factor of $\sim 10$ for methane-dominated planets

\begin{tabular}{|l|l|l|l|}
\hline Property & Requirement & Goal & PFI value \\
\hline Inner Working Angle (IWA) & 0.03 arc sec & & $\begin{array}{l}0.03 \text { arc sec @ } \\
\text { band }\end{array}$ \\
\hline Contrast $(I<8$ mag.) @ IWA & $10^{-8} @ 50$ mas & & $\begin{array}{l}2 \times 10^{-8} \text { without } \\
\text { speckle } \\
\text { suppression }\end{array}$ \\
\hline Contrast $(I<8$ mag.) wide angle & & $10^{-9} @ 100$ mas & \\
\hline Contrast $(H<10$ mag.) @ IWA & $10^{-6} @ 30$ mas & $210^{-1} @ 30$ mas & $\begin{array}{l}1 \times 10^{-6} \text { without } \\
\text { speckle } \\
\text { suppression }\end{array}$ \\
\hline Plate scale & Nyquist @ $H$ & Nyquist @ $J$ & 5.5 mas \\
\hline Field of View (radius) & 0.7 arc sec & 2 arc sec & $2 \times 2$ arc sec \\
\hline Spectral resolution, full FOV & 50 & 100 & 70 \\
\hline Spectral resolution, partial FOV & 500 & 1000 & 700 \\
\hline Wavelength range & $1-2.5 \mu \mathrm{m}$ & $1-4 \mu \mathrm{m}$ & $1-5 \mu \mathrm{m}$ \\
\hline Imaging polarimetry & $\begin{array}{l}\text { Simultaneous } \\
\text { two channel }\end{array}$ & & SDC \\
\hline Sensitivity (1 hr., 5- $\sigma)$ & $H=27$ mag. & $H=32$ mag. & \\
\hline
\end{tabular}

Unless controlled, light scattered by diffraction from the telescope pupil would completely swamp the signal from a planet. After considerable exploration of alternatives using simulations, we have selected a dual-stage shearing nulling interferometer or "nuller" as the diffraction suppression system (DSS), described in Section 4. This combines four offset and phase-shifted copies of the telescope pupil to remove the uniform component of the electromagnetic field that causes diffraction. This has two major advantages over conventional coronagraph architectures. First, it allows for very small inner working angles (IWA) - as small as $3 \lambda / \mathrm{D}$ - that are needed both to detect nearby planets in reflected starlight and to image young solar systems at distances as great as $150 \mathrm{pc}$. Second, it is robust against the large secondary obscurations likely to be found in any extremely large telescope.

The large aperture of TMT allows PFI to exceed the contrast of 8-m ExAO systems by an order of magnitude, but does nothing to relax the fundamental requirement of sub-nanometer internal static optical errors; since the effect of internal errors is partially independent of telescope size, these requirements are even more exacting to reach the $10^{-8}$ contrast levels that we are targeting. To overcome this, we will use a dedicated, interferometric, infrared wavefront sensor that is tightly integrated with the DSS, and controls a second MEMS DM located inside the nuller. Known as the back wavefront sensor, this system is a Mach-Zehnder interferometer combining the bright and dark outputs of the nulling DSS. It has two primary purposes. First, operating at the science wavelength and measuring the dark output of the DSS, it will provide sub-nanometer absolute accuracy and correct these errors through feedback to the front AO or through its 
own MEMS. Second, especially on very red science targets, it will provide additional rejection of atmospheric turbulence, allowing PFI to reach contrast of $10^{-6}$ to $10^{-7}$ on $H=10$ mag. young stars.

PFI's science instrument is a dedicated Integral Field Spectrograph unit (IFS or IFU) optimized for high-contrast imaging, maximum scientific return, and for spectral follow-up of extrasolar planets. This combines moderate spectral resolution $(R=\lambda / \Delta \lambda \sim 70)$ with a $2 \times 2$ arc second Nyquist-sampled field of view and spectral coverage from 1-5 $\mu \mathrm{m}$. Carefully designed to minimize chromatic errors, this allows planets to be distinguished from artifact speckles by their differing behavior as a function of wavelength. The instrument also includes a $R=500$ mode that can be used for followup of previously-detected planets. A dual-channel imaging polarimeter mode will be available to study circumstellar disks and distinguish disk structure from the planets embedded in them. Figure 2 shows a block diagram of the PFI system.

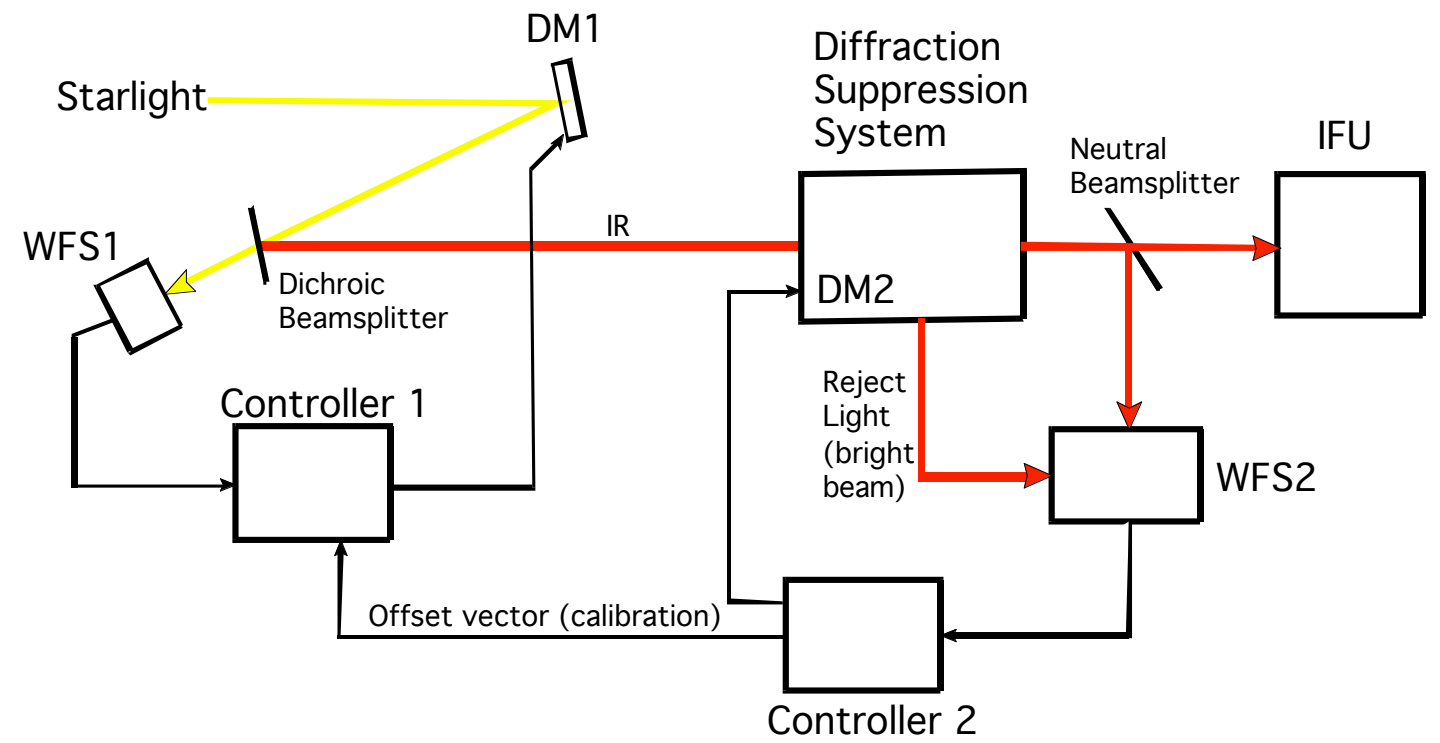

Figure 2: Simplified block diagram of the PFI system.

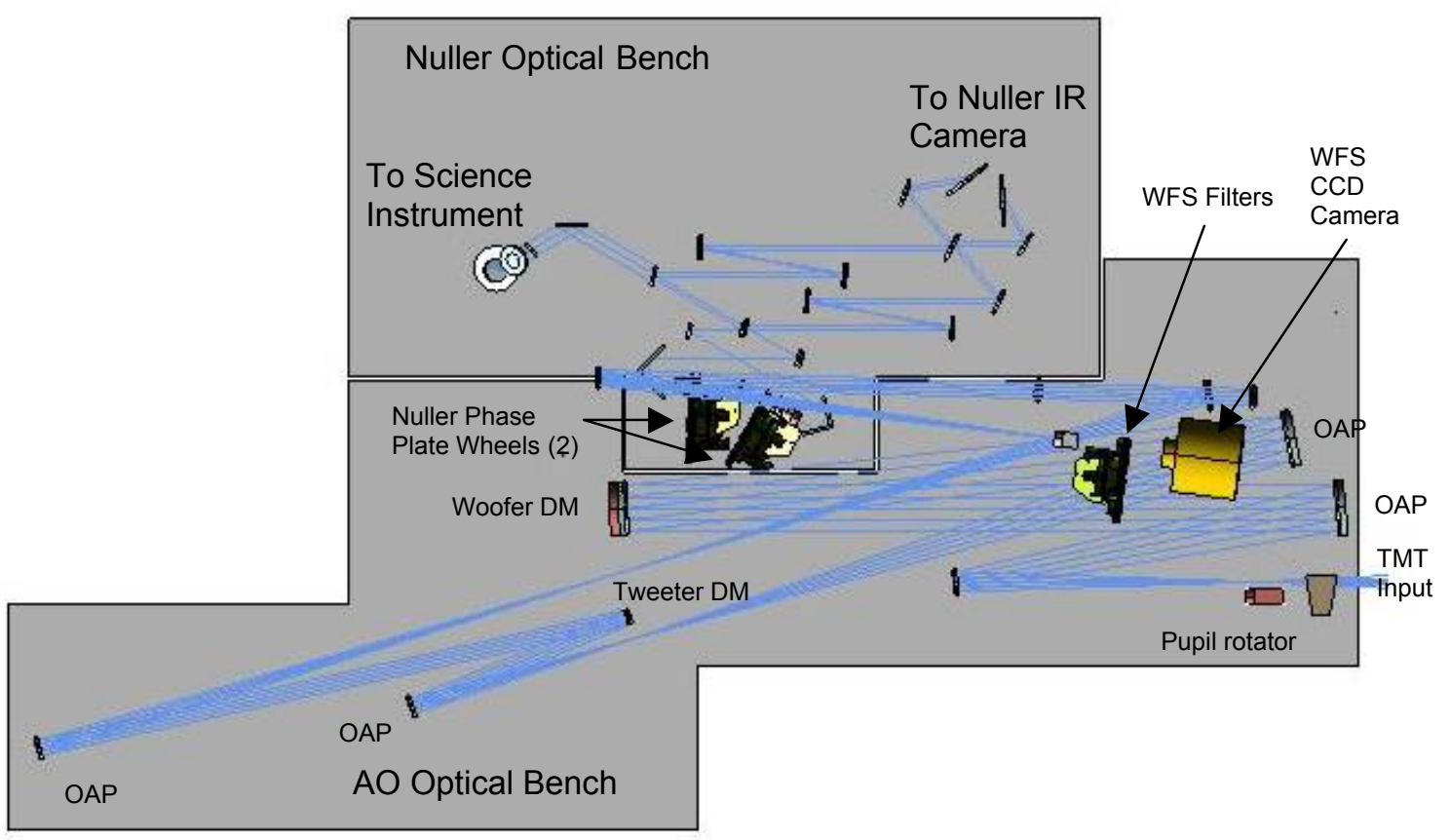

Figure 3: Top-view CAD rendering of the PFI optical bench. Long dimension is $4.5 \mathrm{~m}$ 
Table 2: Properties of PFI compared to the Gemini Planet Imager ${ }^{2}$, an ExAO system for a 8-m telescope

\begin{tabular}{|l|l|l|}
\hline & Gemini Planet Imager & PFI \\
\hline Front WFS & SF Shack-Hartmann & SF Pyramid \\
\hline Primary DM & $\begin{array}{l}64 \times 64 \text { MEMS } \\
(N=48 \text { circle illuminated })\end{array}$ & Two 128x128 \\
\hline Subaperture size & $d=18 \mathrm{~cm}$ & $d=24 \mathrm{~cm}$ \\
\hline Front AO rate & $2500 \mathrm{~Hz}$ & $2000-4000 \mathrm{~Hz}$ \\
\hline Back WFS & IR interferometer & IR interferometer \\
\hline Back WFS rate & $100 \mathrm{~Hz}$ samp. $0.1 \mathrm{~Hz}$ correction & $1000 \mathrm{~Hz}$ correction \\
\hline Limiting magnitude & $I=8 \mathrm{mag}(I=9$ goal $)$ & $H=10 \mathrm{mag}$ \\
\hline Coronagraph IWD & 0.12 arcseconds $($ apodized Lyot $)$ & 0.03 arcsec (nuller $)$ \\
\hline Science instrument & Lenslet IFS & Lenslet IFS \\
\hline Wavelength coverage & $0.9-2.4 \mu \mathrm{m}$ & $1-2.4 \mu \mathrm{m}$ (goal $1-5 \mu \mathrm{m})$ \\
\hline Pixel size & 0.014 arc seconds & 0.005 arcsec \\
\hline Field of view & $3.5 \times 3.5$ arc seconds & $2 \times 2$ arscec \\
\hline Spectral resolution & $\mathrm{R}=40$ & $\begin{array}{l}\mathrm{R}=7020 \% \text { bandwidth } \\
(\mathrm{R}=500 \text { narrowband mode) }\end{array}$ \\
\hline
\end{tabular}

\section{FIRST-STAGE AO SYSTEM}

G5 at $10 \mathrm{pc}$ Mag 4.2, $2029 \mathrm{Ph} / \mathrm{subap}, 2 \mathrm{khz}$

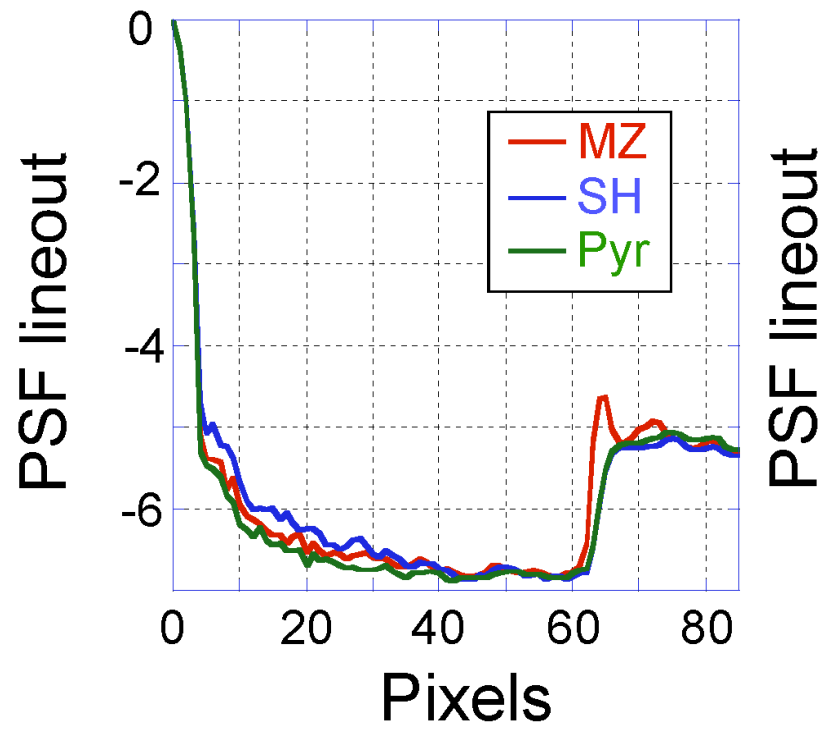

G5 at $30 \mathrm{pc}$ Mag 6.6, $225 \mathrm{Ph} /$ subap, $2 \mathrm{khz}$

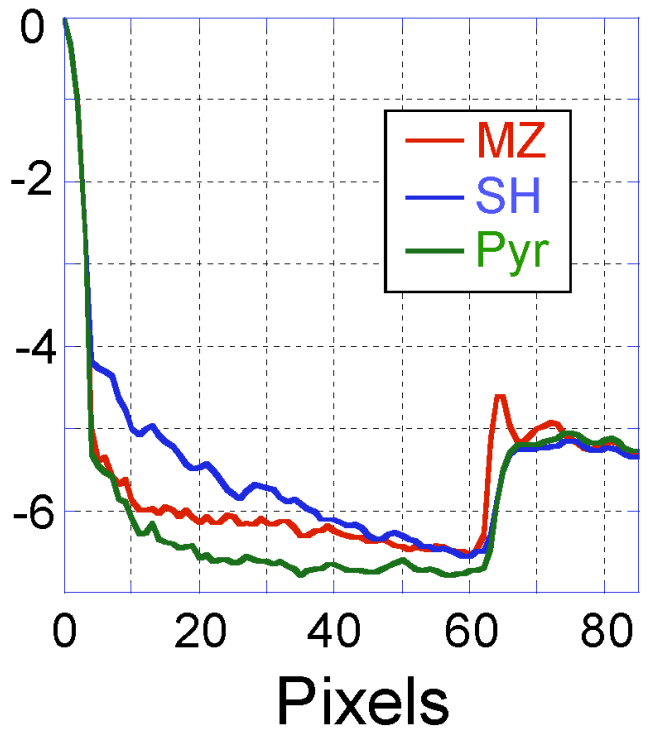

Figure 4: PSF comparison between Shack-Hartmann sensor (top), a Mach-Zehnder interferometer (middle), and the pyramid wavefront sensor (bottom). PSFs have been generated using a simple apodizer rather than a full coronagraph. On bright stars, servo lag dominates and all sensors have similar performance; on dimmer stars, the superior low-frequency measurement of the interferometric sensors can be seen.

As discussed in Section 1, PFI has a two-stage AO system. The initial ("front" or "visible") AO is a very high-speed system operating in visible light $(0.7-1.0 \mu \mathrm{m})$ to provide primary wavefront correction on bright $(I<8 \mathrm{mag})$ targets. On many such targets, servo lags will be the dominant source of scattered light within the dark hole region; as a result, the front AO must be capable of very high frame rates and efficient correction with a limited number of photons per timestep. On dimmer targets, the front-end system provides a modest wavefront correction to increase the Strehl ratio enough to enable the post DSS (or "back") adaptive optics to control the correction of the wavefront. Hence it must also be capable of stable behavior at much lower fluxes $(I<14 \mathrm{mag})$. For this purpose we have selected two possible WFS 
architectures - a pyramid wavefront sensor or an interferometric wavefront sensor - that have much better noise behavior than a conventional Shack-Hartmann sensor. The pyramid sensor is particularly attractive as it has a wide variety of operating modes that remove the need for any additional "bootstrap" sensor over the whole atmospheric turbulence range. Figure 4 compares the performance of a Shack-Hartmann sensor, a Mach-Zehnder interferometer, and the pyramid wavefront sensor.

The interferometric pyramid or ridge wavefront sensor is discussed at length in a companion paper ${ }^{3}$. At low Strehl ratios, a pyramid sensor operates as a slope sensor with high dynamic range; at high Strehl, it begins to operate like an interferometer, measuring phase directly through the interference of the PSF core with individual PSF speckles, with good accuracy and low noise propagation ${ }^{4}$. The pyramid sensor is attractive for a number of reasons. Because this wavefront sensor can be run in both slope mode and interferometric mode, it does not require a separate bootstrapping adaptive optics system as would be required for other interferometric wavefront sensors. In addition because of the geometry of the pyramid sensor, the sub-apertures can be binned on the camera to reduce the number of sub-apertures in the case of low photon flux. Although a classic pyramid sensor divides the PSF into four quadrants, we found better stability in the interferometric mode with a two-sided "ridge" sensor. In this architecture, the PSF is brought to focus on the interface between two lenslets, each of which produces an image of the telescope pupil (Figure 5.) Such a sensor is blind to pure Y phase modes. This can be addressed with a second ridge sensor oriented at right angles to the first. Since the nulling DSS is also blind to planets along one axis, the higher-order Y modes could be ignored.

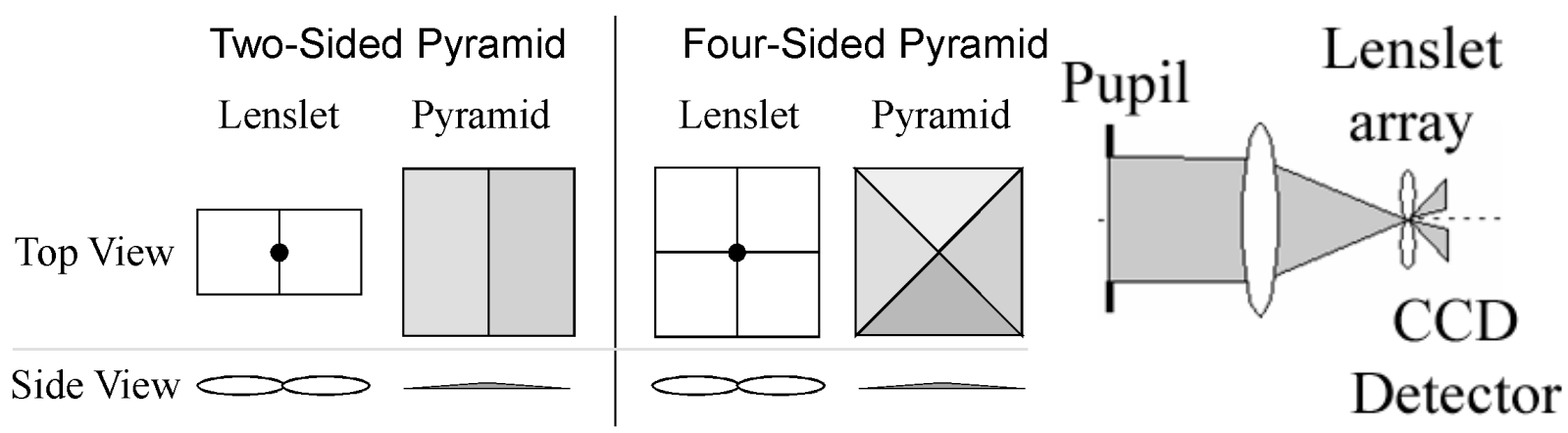

Figure 5: Ridge (two-sided) or pyramid (four-sided) wavefront sensor based on lenslet or pyramid optics.

An alternative to the pyramid sensor is a more conventional interferometer. Interferometric wavefront sensors also measure the wavefront phase directly by interfering reference and signal light. These devices must measure the reference and signal beams within the coherence length of the light, which limits the interferometric designs that can be used with temporally incoherent light from stars. One such design is based on a Mach-Zehnder interferometer ${ }^{5}$. This design must, however, keep the two arms within the coherence length of the light and provide achromatic phase shifts over a modest, $20 \%$, bandwidth. Recently pixilated interferometers have been designed which utilize polarizers to provide achromatic phase shifts ${ }^{6}$ and when placed in a self-referencing configuration automatically maintain the distance between the reference and signal beams forming the interferogram. Although these entail a loss of light due to the polarizers, the robust design makes them attractive for astronomical use.

\subsection{AO technologies for ELT ExAO}

Although ExAO systems on 30-m-class telescopes will necessarily be an order of magnitude more complex than their 8$\mathrm{m}$ counterparts, most necessary technologies seem likely to be in place by the time such an instrument enters preliminary design. The baseline PFI design is optimized for performance on moderate-brightness stars rather than a large outer working angle, so a $128 \times 128$ actuator DM $(d=24 \mathrm{~cm})$ is sufficient. Such DMs are a moderate extrapolation of the $64 \times 64$ DMs under development using MEMS technology or high-density pizeoelectric actuators. Since interferometric wavefront sensors require only one pixel per channel per subaperture, multiple 128x128 CCDs can be used for the visible-light WFS channel; larger-format devices would allow for multiple channels on a single detector. CCDs that combine low noise and high frame rates are under development. ${ }^{7}$ Computational requirements for the Pyramid WFS are high but feasible; we have identified a strawman computer design using DSPs and Field-programmable Gate Arrays 
(FPGAs) that would achieve 1600 frames per second with currently available components - a moderate improvement in computational hardware should allow us to reach our $4 \mathrm{kHz}$ goals.

By using the visible WFS for the fastest $(2-4 \mathrm{kHz})$ atmospheric correction, the requirements on the IR post-DSS WFS are relaxed. However, these requirements are still challenging: an IR array with $\sim 0.5-1 \mathrm{kHz}$ frame rates, $<5$ electrons noise, and $>128 \times 128$ format.

\section{DIFRACTION SUPPRESSION SYSTEM}

Light is scattered within the PSF of a telescope both by wavefront errors and diffraction. Even in the absence of wavefront errors, e.g. a perfect circular telescope in space, diffraction will produce an Airy pattern much brighter than any potential planets. For TMT, the maze of segment and secondary structures within the pupil will produce a brighter and more complex pattern. Although in principal these patterns are static and could be removed through PSF subtraction, the added light results in added photon noise. More significantly, the Airy pattern amplifies speckles caused by dynamic and static wavefront errors (e.g., Soummer and Claude 2004) through the "pinned speckle" cross-terms (Perrin et al. 2003) and, since these speckles are particularly sensitive to PSF shears, reduces the stability of the PSF. This effect is even more significant for TMT, where telescope primary mirror phase errors have the same characteristic $\lambda / d_{\text {seg }}$ spatial frequency characteristics as the telescope diffraction pattern.
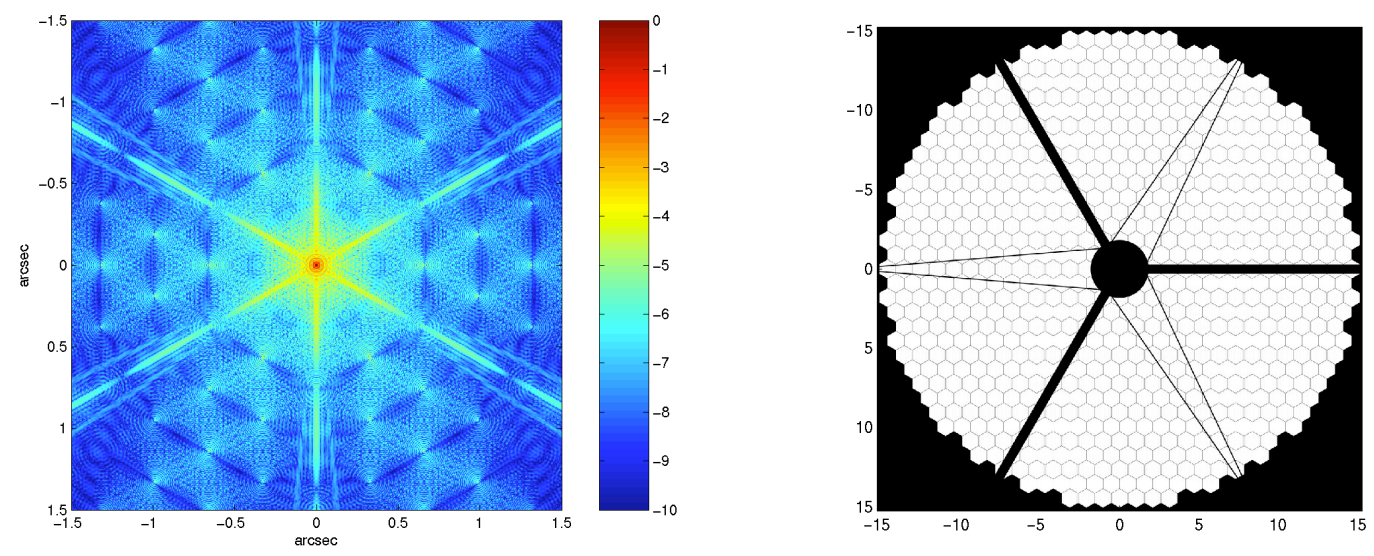

Figure 6: Monochromatic diffraction pattern (PSF) from the Thirty Meter Telescope reference design (left) and the corresponding pupil (right).

PFI therefore requires a Diffraction Suppression System (DSS) that removes diffraction down to levels comparable to the final contrast goals (residual diffraction average intensity $\sim 10^{-8}$ of the peak stellar intensity). It must also allow observations at inner working angles as small as $3 \lambda / \mathrm{D}$. For a segmented telescope with a large secondary obscuration this is an extremely challenging requirement.

During the PFI design study, we analyzed several possible coronagraph architectures before finally selecting a shearing nulling interferometer ${ }^{8}$. We present here the basic design of the nuller, and some performance comparisons with a classic band-limited Lyot coronagraph ${ }^{9}$. In this application, the BLC is strongly limited in its inner working angle due to the secondary-support obscurations in the pupil. (The segment gaps, by contrast, are much less important; for the base TMT gap size of $4 \mathrm{~mm}$ they scatter a small amount of light to very large angles. If necessary, a very mild apodization function can almost completely mitigate the effects of the gaps.) Even with an aggressive Lyot stop with only $10 \%$ throughput performance is mediocre (Figure 7) unless the inner working angle is relaxed to $9 \lambda / \mathrm{D}$. 


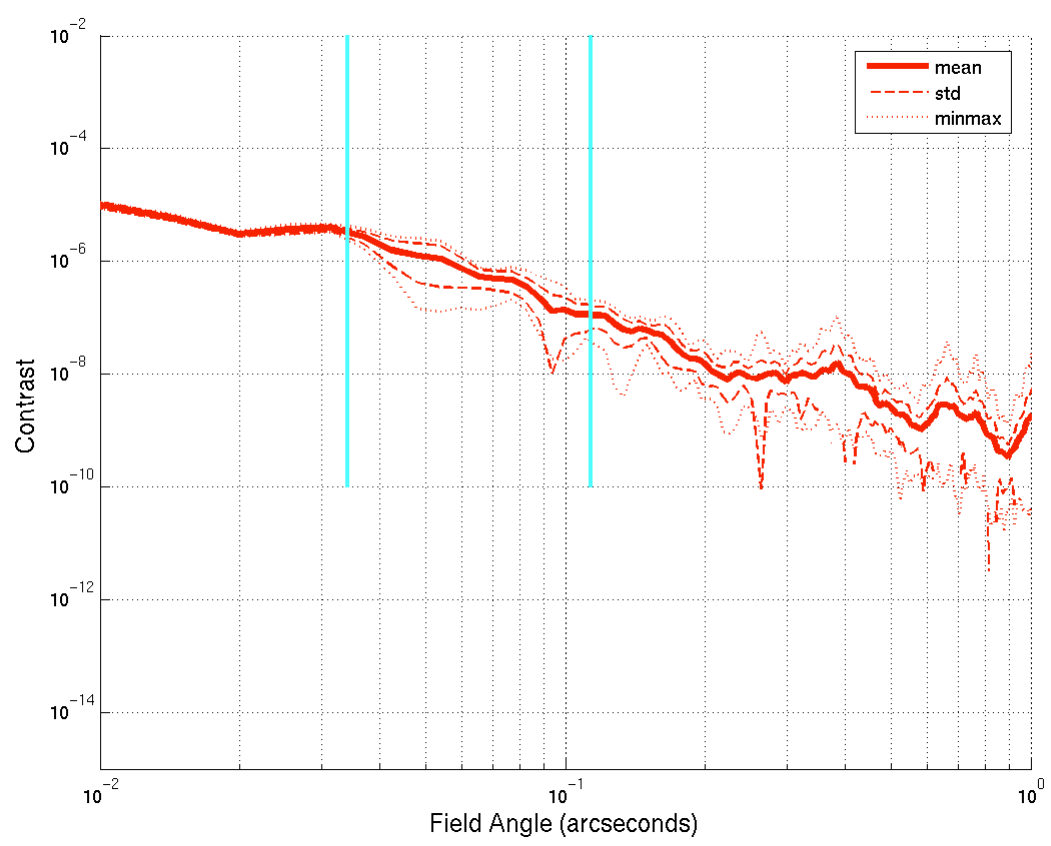

Figure 7: Contrast vs angle for a band-limited coronagraph with a $3 \lambda / \mathrm{D}$ FWHM occultor and a $10 \%$ throughput Lyot stop.

The "visible nuller" shearing nulling interferometer is the baseline we have selected for PFI. The nuller is a variant of a sheared pupil interferometer. In this type of interferometer, the pupil is interfered with a shifted copy of itself. The resulting interferometer beam pattern is a harmonic with a fringe frequency of $B / \lambda$ (cycles/radian) where $B$ is the shear. The nuller implementation of this type of interferometer adds an achromatic $\pi$ phase shift to the field in one of the pupils before recombination. In principle, the field from an on-axis source is perfectly cancelled in the overlap region of the two pupils. Adding multiple shears can decrease the sensitivity to residual tip/tilt errors and the finite size of the target star. Figure 11 shows an optical layout for a single-stage nuller and Figure 8 shows the residual electric field in the pupil plane after a two-stage nuller optimized for small IWD. The secondary obscurations can be easily masked. By tuning the shear to match the segment size the segment gap effects can also be nulled out if necessary, but simulations show that even if they are not cancelled or masked they affect contrast at only the $10^{-9}$ level over most of the field of view. The nuller does have a complicated response to sources on the sky; instantaneously, planets whose angular offset in the X direction is a multiple of $\lambda / \mathrm{B}$ will not be detected. Over the course of a long exposure, though, siderial rotation, deliberate rotation of the instrument/null, and changes to the shear can allow most of the field of view to be covered (Figure 9.) 


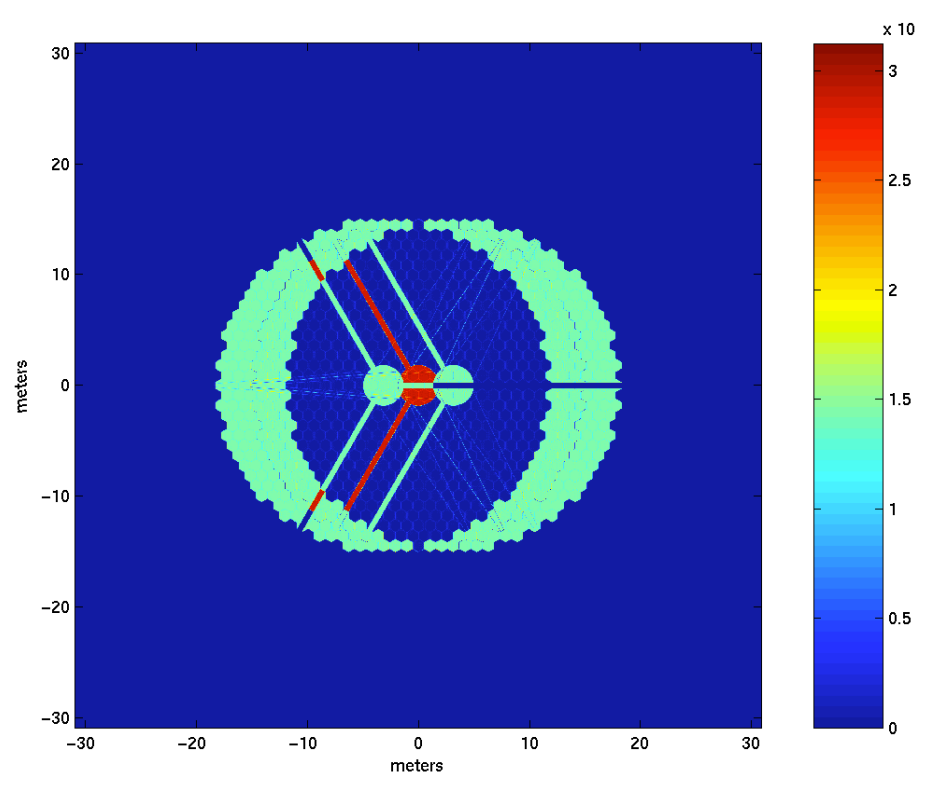

Figure 8: Residual electric field in the pupil plane after a two-stage shearing interferometer.
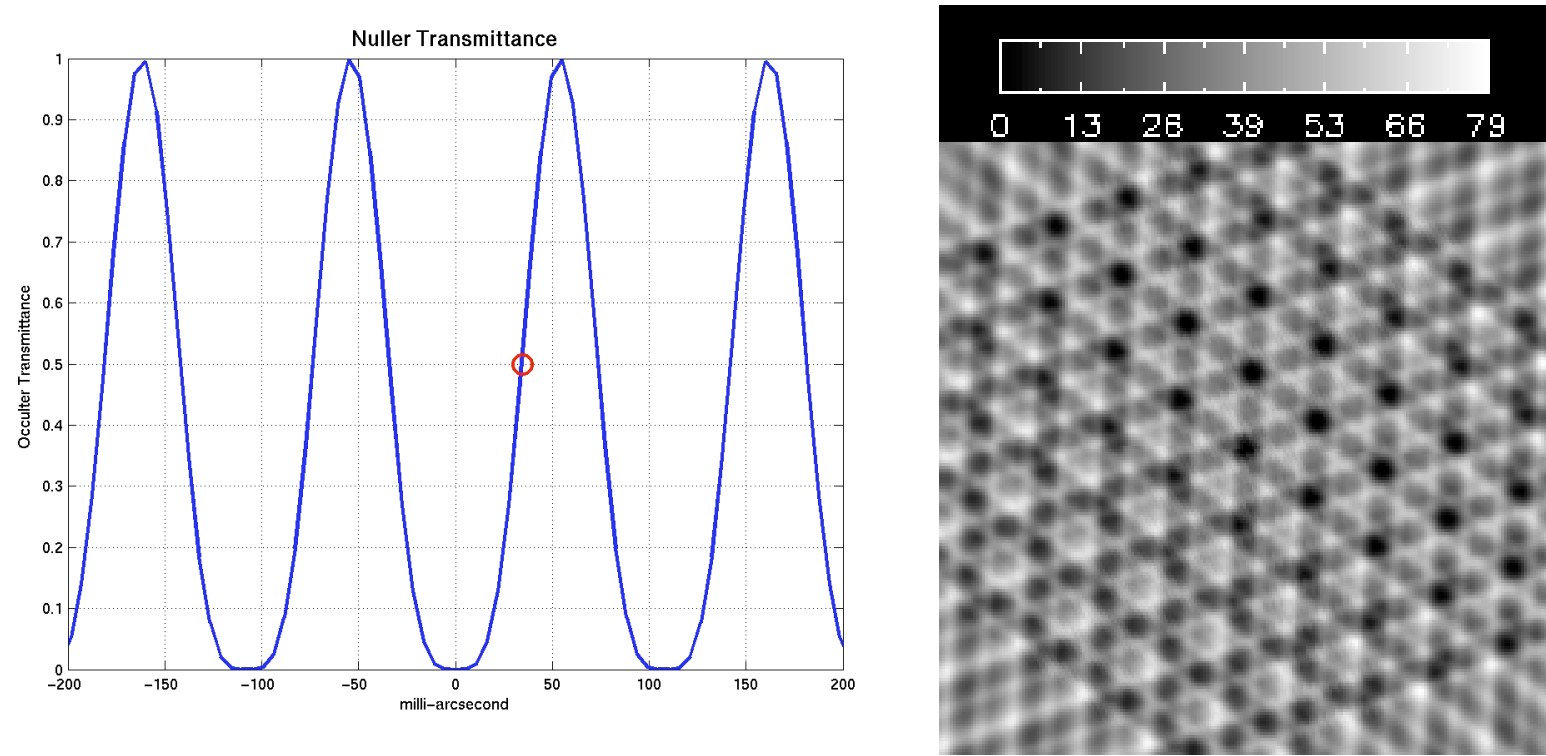

Figure 9: Left: Instantaneous transmission of the nuller as a function of $X$ position in the field of view. Right: average transmission over a 1.2x1.2 arcsecond field of view in a 2-hour exposure in which the field of view has been rotated two times by 120 degrees in addition to siderial rotation. $80 \%$ of the field has at least $50 \%$ transmission.

\section{POST-DSS WFS}

Small wavefront errors due to chromatic effects, changes in non-common-path optics, calibration errors, etc., produce quasi-static speckles that can completely swamp the signal from a planet. To achieve $10^{-8}$ contrast with PFI we require these errors to be below $0.5 \mathrm{~nm}$ over the controlled range of spatial frequencies - an extremely challenging goal. To achieve this, we will integrate a second high-precision IR wavefront sensor into the DSS. This approach is similar to that proposed for the Gemini Planet Imager ${ }^{2}{ }^{10}$, but with an additional goal of operation at $\sim 1 \mathrm{kHz}$ frame rates to allow the IR WFS to provide most of the atmospheric correction on red targets (e.g. T Tauri stars) that are too dim for a good visiblelight pyramid sensor measurement. Rather than feeding instructions from the IR WFS to the initial DM, we have chosen to control a second DM located inside the nuller itself; this allows cancellation of errors in the nuller arms and operation 
in a two-stage controller with significantly better rejection of low temporal-frequency errors. Figure 10 shows a schematic of the post-DSS system. Figure 11 shows an optical design for the nuller and post-DSS WFS.

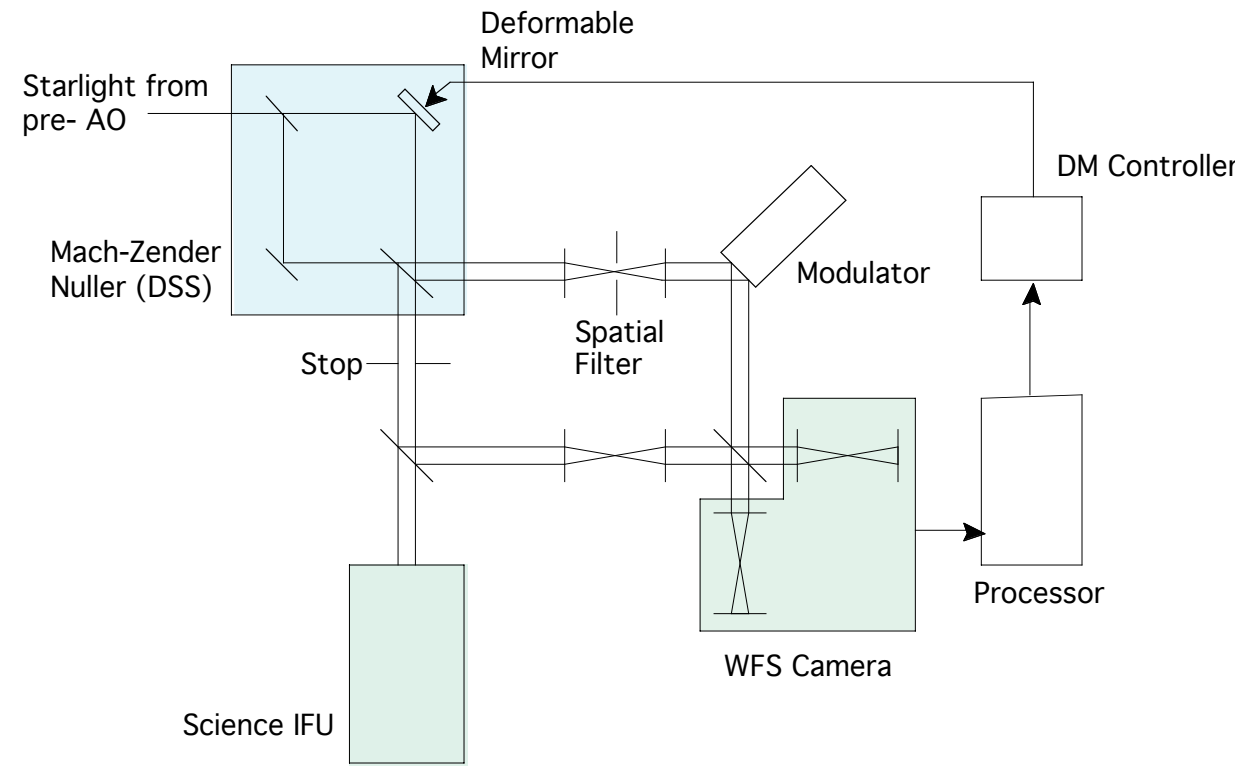

Figure 10: A functional schematic of the backend AO system. A shearing MZ Nuller provides a nulled and a bright output to the backend. The nulled output is split 50:50 between the Science Camera and the post-DSS AO. The bright output is spatially filtered, modulated and interfered with the nulled output at the main beamsplitter. In this case, the combined pupil emergent on both sides of the beamsplitter is reimaged onto a focal plane array. The processor acquires the WFS data and generates a phase map error, which is handed of a controller to close the loop with a DM which is placed in one arm of the nuller.

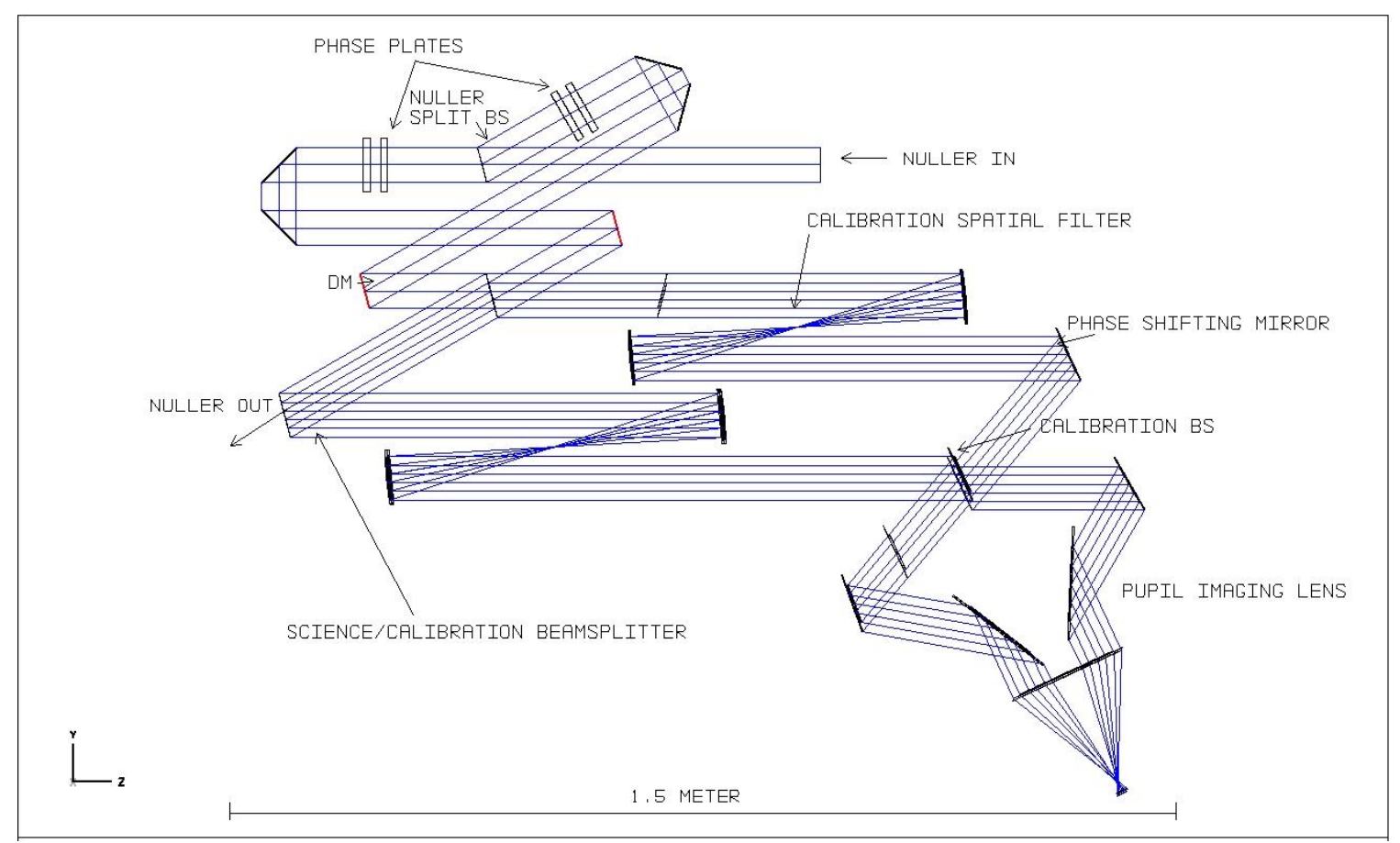

Figure 11. Shown above is a diagram of the PFI nuller and post-DSS calibration WFS unit highlighting the location of key optical elements in the system. Light enters the system from the top right and exits to the science camera at the bottom left. This design shows only a single-stage nuller; for a dual-stage nuller we prefer, a beamsplitter would bring the beam out of the plane into a duplicate nulling interferometer. 


\section{IFS}

The primary purposes of the PFI science instrument are to detect planetary companions by distinguishing them from PSF speckle noise, to record low- and medium resolution 1-2.5 $\mu \mathrm{m}$ spectra of these planets, and to detect and measure circumstellar disks, particularly through polarization. Two speckle-noise suppression techniques are used depending on the science applications. The first one consists of acquiring multi-wavelength image cubes simultaneously and taking advantage of the deterministic behavior of the speckle pattern with wavelength for discriminating speckles from a true companion signal, i.e. the position of interference speckles in the focal plane scales with linearly with wavelength, and hence broad-band speckles appear elongated compared to a point source companion. The second technique is to take advantage of the polarized nature of the companion/disk to distinguish it from the unpolarized speckle pattern of the central star.

Experience with the three-channel camera TRIDENT on CFHT $^{11}$ has shown that the speckle-suppression performance depends critically on the detailed implementation of the instrument. In particular we must minimize the presence of noncommon path aberrations between different wavelength channels (NCA) in the optical system. In the regime where performances are speckle-noise limited, a useful figure of merit for the speckle-suppression performance is simply the speckle-noise attenuation factor $\tau \equiv N / \Delta N$ where $N$ is the input PSF noise on the science instrument and $\Delta N$ is the residual noise left after data processing. One is striving to make $\tau$ as high as possible to subtract speckles down to the photon noise limit without affecting the signal (spectrum) of the companion.

The science instrument is required not only to achieve the highest speckle-noise attenuation but also to yield the best possible throughput, the largest FOV and spectral resolution. Some compromises are inevitable to meet all these criteria. The most attractive solution is a lenslet-based IFS such as the OSIRIS instrument ${ }^{12}$. The lenslet architecture is particularly robust against optical errors in the dispersed beam. Laboratory measurements with a prototype lenslet IFS optimized for speckle suppression are reported elsewhere in this conference. ${ }^{13}$

Table 3: Properties of the PFI science instrument.

\begin{tabular}{|l|l|}
\hline Detector & $4096 \mathrm{x} 4096$ Hawaii4-RG \\
\hline Detector pitch & $10 \mu \mathrm{m}$ \\
\hline Fore optics focal ratio & $\mathrm{f} / 126$ \\
\hline Collimator focal ratio & $\mathrm{f} / 3$ \\
\hline Camera focal ratio & $\mathrm{f} / 3$ \\
\hline Wavelength range & $1.1-2.4 \mu \mathrm{m}$ \\
\hline Spectral Resolution $\lambda / \Delta \lambda$ & 70 and 500 \\
\hline Field of view & $2.2 " \mathrm{x} 2.2^{\prime \prime}$ \\
\hline Spectral band pass & 0.18 (one atmospheric window \\
& per exposure) in $\mathrm{R}=70$ mode \\
\hline Polarimetric spectral band pass & 0.1 \\
\hline Lenslet sampling $(\mathrm{mas} / \mu$-lens): & 5.15 \\
\hline Lenslet pitch $(\mu \mathrm{m})$ & 95 \\
\hline Lenslet group pattern $(p)$ & 3 \\
\hline
\end{tabular}

\section{EFFECTS OF THE TMT PRIMARY MIRROR}

At this early stage of telescope design, it is particularly crucial to analyze the effects of design choices, such as primary mirror requirements, on the performance of possible science instruments. A companion paper ${ }^{14}$ discusses the effects of the TMT primary mirror in greater detail; the results are summarized here. As discussed above, the diffraction of the segment gaps is a relatively minor effect, as are the telescope secondary and tertiary mirrors. Reflectivity variations and phasing of the individual segments have the largest impact on contrast. Figure 12 shows the effects of these key issues. The largest effect is reflectivity variations. $1 \%$ RMS segment-to-segment random reflectivity variations were simulated to represent aging of segments through a typical aluminization cycle. This would limit instantaneous contrast to the $10^{-7}$ 
level. This can be mitigated through post-processing - such pure-pupil-plane errors are likely to be easy to reject through multiwavelength imaging and PSF subtraction - but likely only to a factor of $\sim 20$ in typical exposures, insufficient to meet the full science goals. To overcome this, PFI will include an additional deformable mirror at a non-pupil conjugate to allow control of both phase and amplitude, using information from the post-DSS WFS.

The next-largest errors are those due to the segment shapes themselves; aberrations on individual segments produce discontinuities at the segment edges. The dash-dot curve in Figure 12 shows these assuming that combined segment phase errors are controlled by warping harnesses set by measurements through a system similar to that used at the Keck Observatory. This level of performance would limit PFI performance at small angles, impacting the study of Doppler planets through their reflected light. If this can be improved through better control algorithms and high-precision measurements (perhaps provided by PFI itself), performance would reach the dotted curve, sufficient for all science goals. The scattered light within the "dark hole" from segment errors is primarily due to high-order "beating" terms that conventional phase conjugation wavefront correction ignores ${ }^{15}$. More sophisticated control algorithms could be developed to partially cancel these speckles over a subset of the field of view.

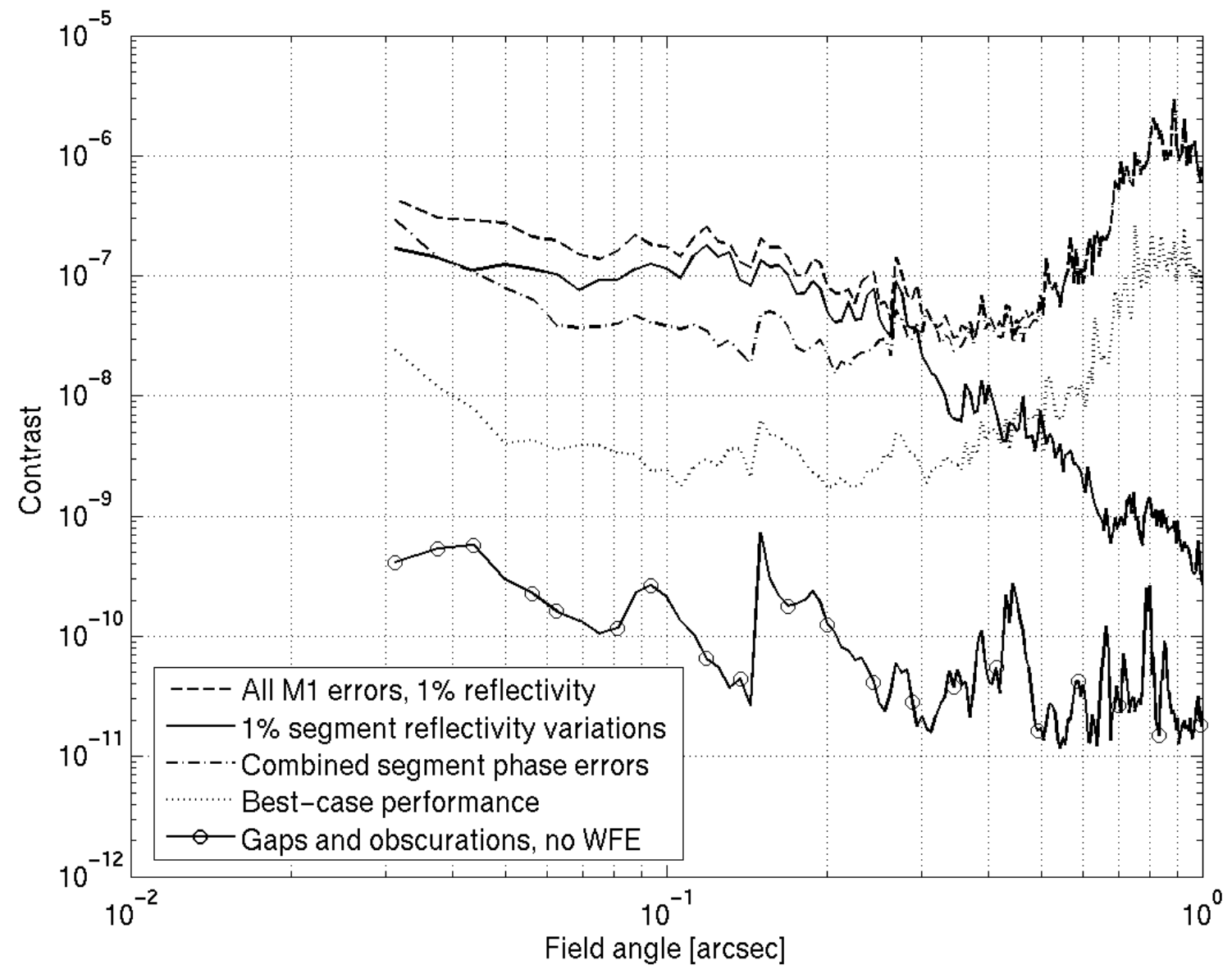

Figure 12: The contrast is shown as a function of field angle. From top to bottom: The dashed curve shows the contrast from all worst-case primary mirrors errors. The solid curve shows the effect of $1 \%$ (RMS) segment-to-segment reflectivity variations. The dash-dot curve shows the contrast assuming uniform reflectivity but individual segment phase errors comparable to the current Keck performance. The dotted curve ("Best case") is the contrast with intensity errors corrected and segment aberrations set by the theoretical performance of TMT warping harnesses. The bottom curve shows the contrast with a perfect TMT telescope. 


\section{PERFORMANCE AND CONCLUSIONS}

We ran a series of end-to-end simulations of PFI incorporating all the subsystems except the science instrument (only monochromatic PSFs were generated.) Typical simulation exposure times were 2-4 seconds, sufficient for 1-4 atmospheric speckle lifetimes ${ }^{16}$. These simulations correctly predict contrast due to atmospheric effects (including scintillation and propagation chromaticity) but are too short to show the effects of static wavefront errors. Separate simulations of static effects were used to evaluate their magnitude and set instrument requirements. If these are met, sensitivity can then be extrapolated to 1-2 hour exposures as shown in Figure 13. Additional post-processing suppression of speckle noise by a factor of 5-10 should be possible.

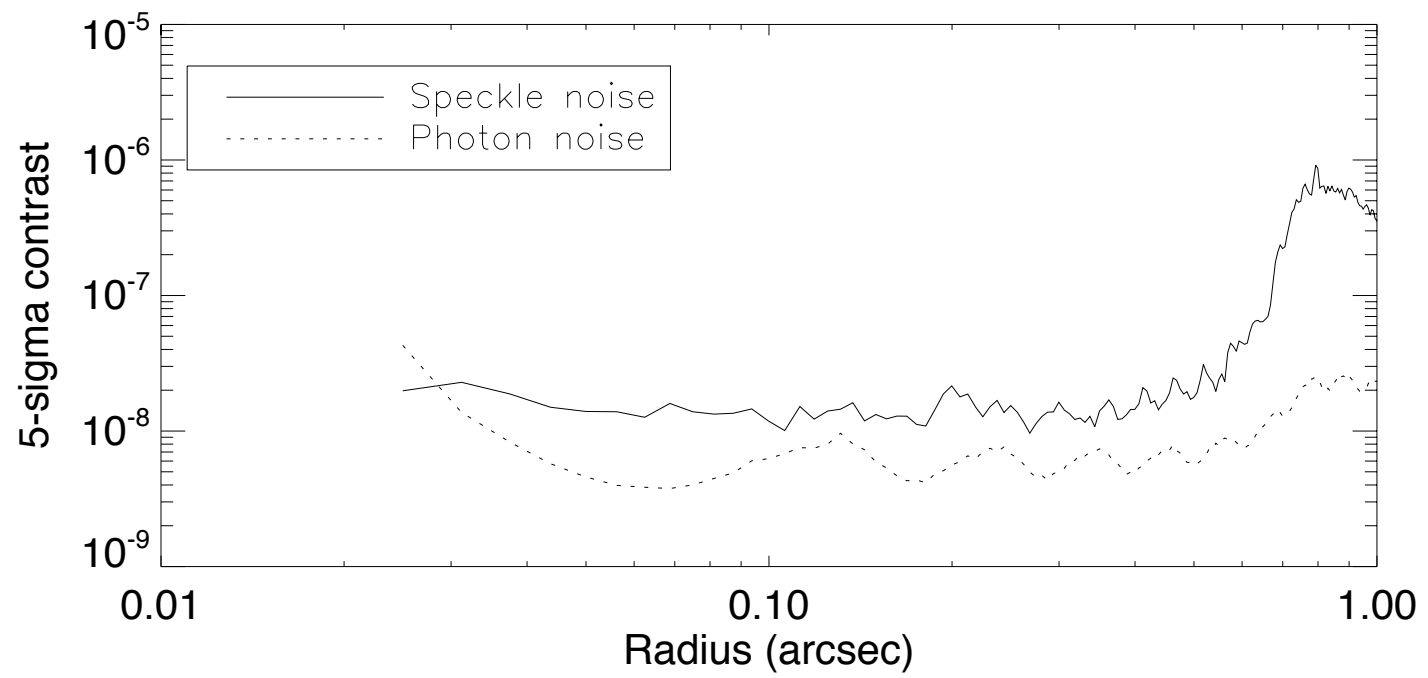

Figure 13: The speckle and photon noise contrast is shown as a function of field angle for a G5 star at 10 pc. The simulation was run for 1.5 seconds and the results scaled to obtain the contrast in $2 \mathrm{hrs}$, assuming systematic errors are controlled.

These results are combined with Monte Carlo models of the solar neighborhood planet population to evaluate PFI's science reach. As an example, Figure 14 shows the population of planets that would be discovered in a "blind" field survey with both PFI and the Gemini Planet Imager (GPI.) As can be seen, PFI has much greater sensitivity to cool, mature planets.

In conclusion, we have identified key science missions - the study of mature planets and of young protoplanets in starforming regions - in which future 30-m-class telescopes will have unique capabilities, in particular their small inner working angle. We have produced a science-driven design, practical using near-term technologies, that can carry out these science missions, with the emphasis on performance at 3-5 $\lambda / \mathrm{D}$. Such an instrument will build upon the technological and scientific heritage of the 8-m class ExAO systems such as the Gemini Planet Imager, and will produce both a uniform census of extrasolar planets and the first images and spectra of planets in the process of formation. 


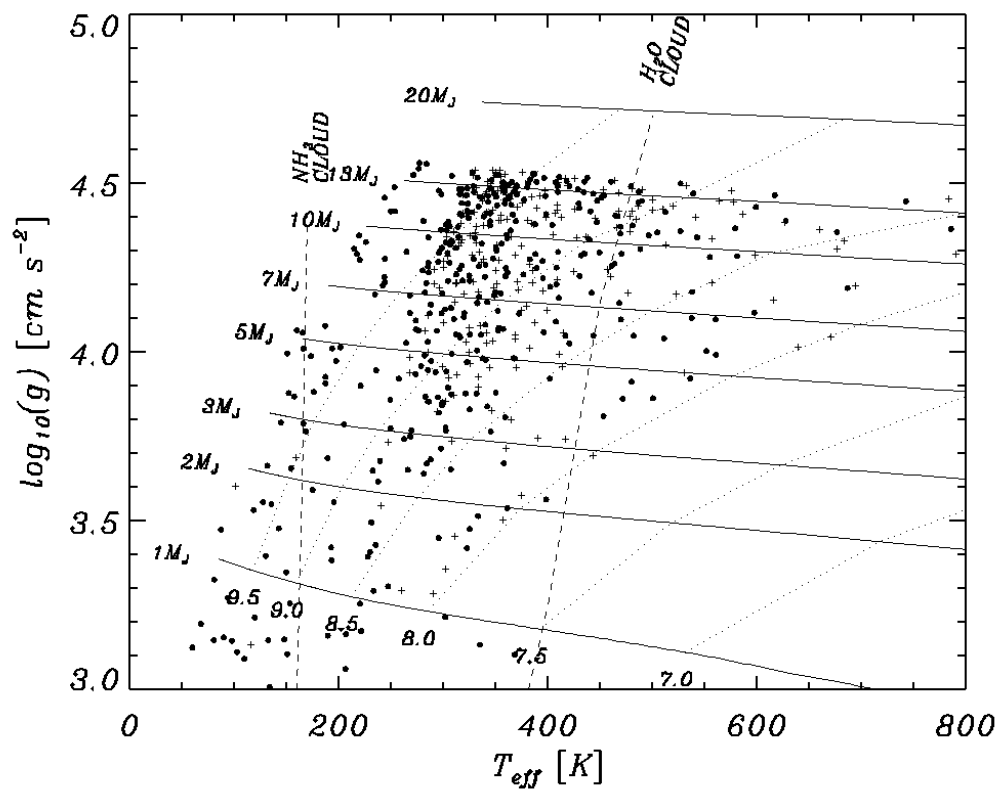

Figure 14: Isochrones [dotted \& labeled with ages in $\log 10(\mathrm{t} / \mathrm{yr})$ ] and iso-mass contours (solid and labeled with masses in MJ) in the surface gravity/effective temperature space for exoplanet atmospheres (data from Burrows et al. 1997) together with the planets discovered by PFI (solid circles) and Gemini Planet Imager (crosses). The water and ammonia cloud condensation lines are shown.

\section{ACKNOWLEDGEMENTS}

This research was performed under the auspices of the U.S. Department of Energy by the University of California, Lawrence Livermore National Laboratory under Contract W-7405-ENG-48, and also supported in part by the National Science Foundation Science and Technology Center for Adaptive Optics, managed by the University of California at Santa Cruz under cooperative agreement No. AST - 9876783. This research was carried out in part at the Jet Propulsion Laboratory, California Institute of Technology, and was sponsored by the California Institute of Technology and the National Aeronautics and Space Administration. The authors gratefully acknowledge the support of the TMT partner institutions. They are the Association of Canadian Universities for Research in Astronomy (ACURA), the Association of Universities for Research in Astronomy (AURA), the California Institute of Technology and the University of California. This work was supported, as well, by the Canada Foundation for Innovation, the Gordon and Betty Moore Foundation, the National Optical Astronomy Observatory, which is operated by AURA under cooperative agreement with the National Science Foundation, the Ontario Ministry of Research and Innovation, and the National Research Council of Canada.

\section{REFERENCES}

1. G. Chauvin et al., "A giant planet candidate near a young brown dwarf. Direct VLT/NACO observations using IR wavefront sensing", $A \& A 425$, L29 (2004)

2. B. Macintosh et al., "The Gemini Planet Imager”, Proc. SPIE 6272 (this volume) (2006)

3. Phillion, D., and Baker, K., "Two-Sided Pyramid Wavefront Sensor in the Direct Phase Mode", Proc. SPIE 6272 (this volume) (2006) 
4. Verinaud, C., "On the nature of the measurements provided by a pyramid wavefront sensor," Opt. Comm. 233, 27-38, (2004)

5. Angel, J. R. P., "Ground-based imaging of extra solar planets using adaptive optics," Nature 368, 203-207, (1994)

6. Millerd, J., J. Hayes, M. North-Morris, M. Novak, and J. Wyant, "Pixellated Phase-Mask Dynamic Interferometer," Proc. SPIE 5531, 304, 2004

7. Hartmann, R., et al., "Results of a fast pnCCD detector system", Proc. SPIE 5903, 210 (2005)

8. Mennesson, B., et al., "Optical Planet Discoverer”, Proc. SPIE 4860, 32 (2003)

9. Kuchner, M., and Traub, W., “A coronagraph with a band-limited mask for finding terrestrial planets”, Ap.J. 570, 900 (2002)

10. Wallace, J.K., et al., "Science camera calibration for extreme adaptive optics", Proc. SPIE 5490, 370 (2004)

11. Marois, C., et al., "TRIDENT: An Infrared Differential Imaging Camera Optimized for the Detection of Methanated Substellar Companions", PASP 117, 745 (2005)

12. Larkin, J., et al., "OSIRIS: Integral field spectrograph for the Keck adaptive optics system”, Proc. SPIE 4841, 1600 (2003)

13. Lavigne, J-F., et al., "An infrared integral field spectrograph specialized for speckle suppression and the detection of extrasolar planets", Proc. SPIE 6269 (2006)

14. Troy, M., et al, "Effects of Diffractoin and Static Wavefront Errors on High-Contrast Imaging from the Thirty Meter Telescope", Proc. SPIE 6272 (2006)

15. Give'on, A., Kasdin, N.J., Vanderbei, R.J., and Avitzour, Y., "On representing and correcting wavefront errors in high-contrast imaging systems," J. Opt. Soc. Am. A 23, 1063 (2006)

16. Macintosh, B., et al., "Speckle lifetimes in high-contrast adaptive optics", Proc. SPIE 5903, 170 (2005) 